\title{
On the complexity of some colorful problems parameterized by treewidth
}

Fellows, Michael R.; Fomin,, Fedor V.; Lokshtanov, Daniel; Rosamond, Frances; Saurabh, Sakel; Szeider, Stefan; Thomassen, Carsten

Published in:

Information and Computation

Link to article, DOI:

10.1016/j.ic.2010.11.026

Publication date:

2011

Document Version

Publisher's PDF, also known as Version of record

Link back to DTU Orbit

Citation (APA):

Fellows, M. R., Fomin, F. V., Lokshtanov, D., Rosamond, F., Saurabh, S., Szeider, S., \& Thomassen, C. (2011). On the complexity of some colorful problems parameterized by treewidth. Information and Computation, 209(2), 143-153. https://doi.org/10.1016/j.ic.2010.11.026

\section{General rights}

Copyright and moral rights for the publications made accessible in the public portal are retained by the authors and/or other copyright owners and it is a condition of accessing publications that users recognise and abide by the legal requirements associated with these rights.

- Users may download and print one copy of any publication from the public portal for the purpose of private study or research.

- You may not further distribute the material or use it for any profit-making activity or commercial gain

- You may freely distribute the URL identifying the publication in the public portal 
Provided for non-commercial research and education use. Not for reproduction, distribution or commercial use.

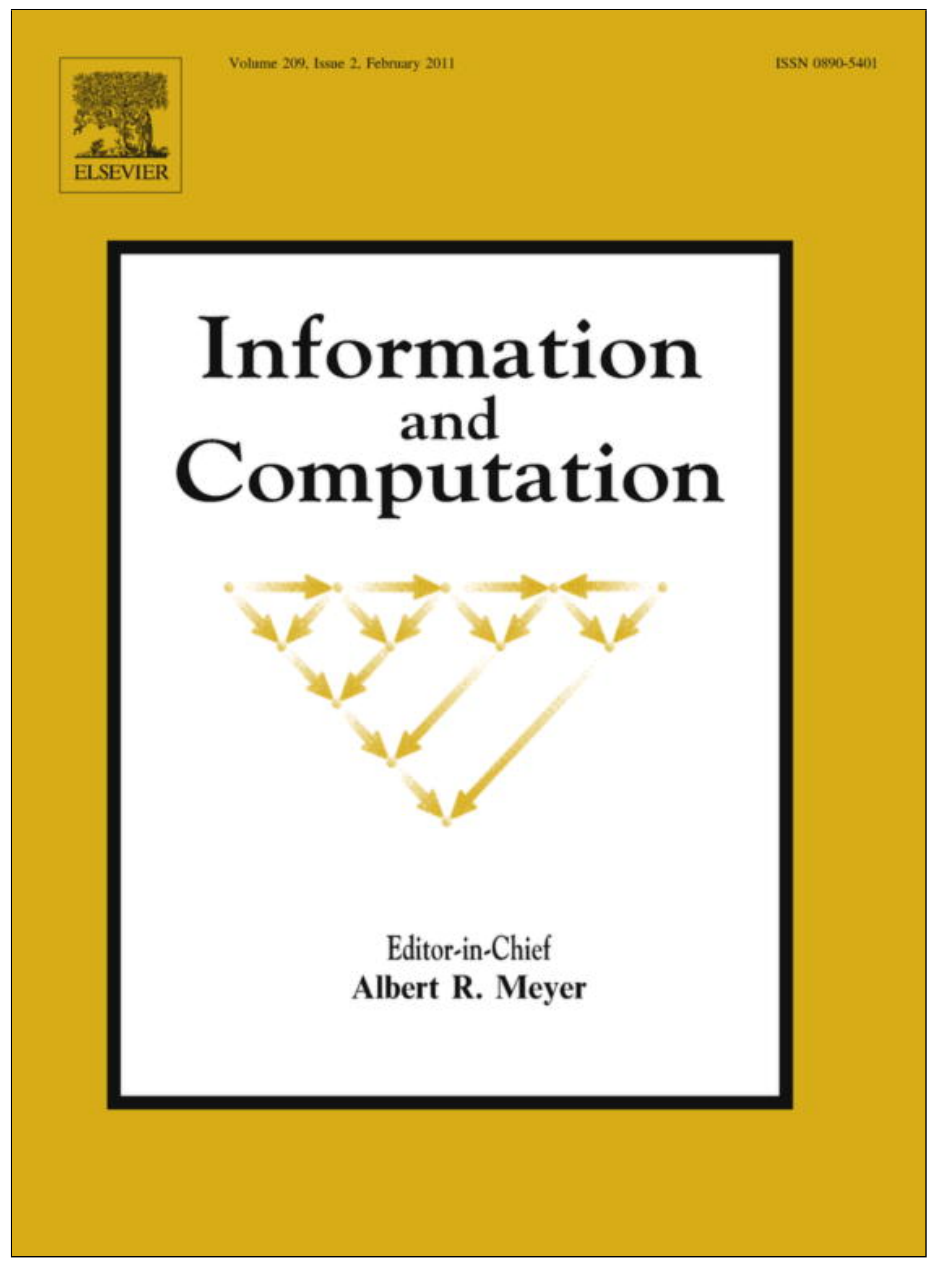

This article appeared in a journal published by Elsevier. The attached copy is furnished to the author for internal non-commercial research and education use, including for instruction at the authors institution and sharing with colleagues.

Other uses, including reproduction and distribution, or selling or licensing copies, or posting to personal, institutional or third party websites are prohibited.

In most cases authors are permitted to post their version of the article (e.g. in Word or Tex form) to their personal website or institutional repository. Authors requiring further information regarding Elsevier's archiving and manuscript policies are encouraged to visit:

http://www.elsevier.com/copyright 


\title{
On the complexity of some colorful problems parameterized by treewidth ${ }^{\text {th }}$
}

\author{
Michael R. Fellows ${ }^{\mathrm{a}}$, Fedor V. Fomin ${ }^{\mathrm{b}, *}$, Daniel Lokshtanov ${ }^{\mathrm{c}}$, Frances Rosamond ${ }^{\mathrm{a}}$, \\ Saket Saurabh ${ }^{\mathrm{d}}$, Stefan Szeider ${ }^{\mathrm{c}}$, Carsten Thomassen ${ }^{\mathrm{f}}$ \\ a Charles Darwin University, Darwin, Australia \\ $\mathrm{b}$ Department of Informatics, University of Bergen, Bergen, Norway \\ c Department of Computer Science and Engineering, University of California, USA \\ $\mathrm{d}$ The Institute of Mathematical Sciences, Chennai, India \\ e Institute of Information Systems, Vienna University of Technology, Vienna, Austria \\ f Mathematics Institute, Danish Technical University, Lyngby, Denmark
}

\section{A RTICLE IN F O}

\section{Article history:}

Received 7 September 2009

Revised 29 July 2010

Available online 29 November 2010

\section{Keywords:}

Parameterized complexity

Bounded treewidth

Graph coloring

\begin{abstract}
A B S T R A C T
In this paper, we study the complexity of several coloring problems on graphs, parameterized by the treewidth of the graph.

1. The List Coloring problem takes as input a graph $G$, together with an assignment to each vertex $v$ of a set of colors $C_{v}$. The problem is to determine whether it is possible to choose a color for vertex $v$ from the set of permitted colors $C_{v}$, for each vertex, so that the obtained coloring of $G$ is proper. We show that this problem is $W[1]$-hard, parameterized by the treewidth of $G$. The closely related PrEColoring ExTENSION problem is also shown to be $W[1]$-hard, parameterized by treewidth.

2. An equitable coloring of a graph $G$ is a proper coloring of the vertices where the numbers of vertices having any two distinct colors differs by at most one. We show that the problem is hard for $W[1]$, parameterized by the treewidth plus the number of colors. We also show that a list-based variation, LIST EQUITABLE COLORING is W[1]-hard for forests, parameterized by the number of colors on the lists.

3. The list chromatic number $\chi_{l}(G)$ of a graph $G$ is defined to be the smallest positive integer $r$, such that for every assignment to the vertices $v$ of $G$, of a list $L_{v}$ of colors, where each list has length at least $r$, there is a choice of one color from each vertex list $L_{v}$ yielding a proper coloring of $G$. We show that the problem of determining whether $\chi_{l}(G) \leq r$, the LIST Chromatic Number problem, is solvable in linear time on graphs of constant treewidth. (c) 2010 Elsevier Inc. All rights reserved.
\end{abstract}

\section{Introduction}

It is well-known that many computationally hard problems are solvable in polynomial time when the input is an $n$-vertex graph $G$ of treewidth $t$. Whether the running time of the algorithm is of the form $n^{O(t)}$ or $f(t) \cdot n^{O(1)}$ differs from problem to problem, and this drastically affects the practical applicability of the algorithm. In this paper, we initiate the systematic classification of problems on graphs on bounded treewidth based on whether there exists an algorithm for the problem with running time $f(t) \cdot n^{O(1)}$. In particular we consider the complexity of various graph coloring problems, for graphs of bounded treewidth, in the framework of parameterized complexity $[10,12,26]$, where we take the parameter to be the treewidth $t$. For the definition of the graph invariant treewidth and related concept we refer to [3].

\footnotetext{
theliminary results of this paper appeared in [13].

* Corresponding author.

E-mail addresses: michael.fellows@cdu.edu.au (M.R. Fellows), fomin@ii.uib.no, Fedor.Fomin@ii.uib.no (F.V. Fomin), daniello@ii.uib.no (D. Lokshtano), frances.rosamond@cdu.edu.au (F. Rosamond), saket@imsc.res.in (S. Saurabh), stefan@szeider.net (S. Szeider), C.Thomassen@mat.dtu.dk(C. Thomassen).
} 
Coloring problems that involve local or global restrictions on the coloring have many important applications in such areas as operations research, scheduling and computational biology, and also have a long mathematical history. For recent surveys of the area one can turn to [1,23,29,31] and also the book [21] and the Ph.D. dissertation of Marx [24].

\title{
1.1. Problems considered
}

A proper coloring of a graph is an assignment of colors to its vertices such that the endpoints of every edge get distinct colors (that is no edge is monochromatic). Similarly, a proper $q$-coloring of graph $G=(V, E)$ is an assignment of colors from $\{1,2, \ldots, q\}$ to the vertices of $G$ such that no edge is monochromatic. The chromatic number of a graph $G$, denoted by $\chi(G)$, is the minimum $q$ for which a proper $q$-coloring exists. The list chromatic number $\chi_{l}(G)$ of a graph $G$ is defined to be the smallest positive integer $r$, such that for every assignment to the vertices $v$ of $G$, of a list $L_{v}$ of colors, where each list has length at least $r$, there is a choice of one color from each vertex list $L_{v}$ yielding a proper coloring of $G$. Next we define all the main problems we consider in this paper.

\author{
LIST COLORING \\ Input: A graph $G=(V, E)$ of treewidth at most $t$, and for each vertex $v \in V$, a list $L_{v}$ of permitted colors. \\ Parameter: $t$ \\ Question: Is there a proper vertex coloring $c$ with $c(v) \in L_{v}$ for each $v$ ?
}

\section{PRECOLORING EXTENSION}

Input: A graph $G=(V, E)$ of treewidth at most $t$, a subset $W \subseteq V$ of precolored vertices, a precoloring $c_{W}$ of the vertices of $W$, and a positive integer $r$.

Parameter: $t$

Question: Is there a proper vertex coloring $c$ of $V$ which extends $c_{W}$ (that is, $c(v)=c_{W}(v)$ for all $v \in W$ ), using at most $r$ colors?

\section{EQuitable Coloring (ECP)}

Input: A graph $G=(V, E)$ of treewidth at most $t$ and a positive integer $r$.

Parameter: $t$

Question: Is there a proper vertex coloring $c$ using at most $r$ colors, with the property that the sizes of any two color classes differ by at most one?

\section{LIST CHROMATIC NUMBER}

Input: A graph $G=(V, E)$ of treewidth at most $t$, and a positive integer $r$.

Parameter: $t$

Question: Is $\chi_{l}(G) \leq r$ ?

\subsection{Framework of study - background on parameterized complexity}

We obtain all our results in the framework of parameterized complexity. Here, we provide some essential background of the theory. Parameterized complexity is basically a two-dimensional generalization of "P vs. NP" where in addition to the overall input size $n$, one studies the effects on computational complexity of a secondary measurement that captures additional relevant information. This additional information can be, for example, a structural restriction on the input distribution considered, such as a bound on the treewidth of an input graph. Parameterization can be deployed in many different ways; for general background on the theory see $[10,12,26]$.

The two-dimensional analogue (or generalization) of $\mathrm{P}$, is solvability within a time bound of $O\left(f(k) n^{c}\right)$, where $n$ is the total input size, $k$ is the parameter, $f$ is some (usually computable) function, and $c$ is a constant that does not depend on $k$ or $n$. Parameterized decision problems are defined by specifying the input, the parameter, and the question to be answered. A parameterized problem that can be solved in such time is termed fixed-parameter tractable (FPT). There is a hierarchy of intractable parameterized problem classes above FPT, the main ones are:

$$
F P T \subseteq M[1] \subseteq W[1] \subseteq M[2] \subseteq W[2] \subseteq \cdots \subseteq W[P] \subseteq X P
$$

The principal analogue of the classical intractability class NP is $W[1]$, which is a strong analogue, because a fundamental problem complete for $W[1]$ is the $k$-STEP HALTING Problem fOR NondeTERMinistic TURING MachinEs (with unlimited nondeterminism and alphabet size) - this completeness result provides an analogue of Cook's Theorem in classical complexity. A convenient source of $W[1]$-hardness reductions is provided by the result that $k$-CLIQUE is complete for $W[1]$. Other highlights of the theory include that $k$-DominATING SET, by contrast, is complete for $W[2]$. FPT $=M[1]$ if and only if the Exponential Time Hypothesis fails. XP is the class of all problems that are solvable in time $O\left(n^{g(k)}\right)$.

The principal "working algorithmics" way of showing that a parameterized problem is unlikely to be fixed-parameter tractable is to prove $W[1]$-hardness. The key property of a parameterized reduction between parameterized problems $\Pi$ 
and $\Pi^{\prime}$ is that the input $(x, k)$ to $\Pi$ should be transformed to input $\left(x^{\prime}, k^{\prime}\right)$ for $\Pi^{\prime}$, so that the receiving parameter $k^{\prime}$ is a function only of the parameter $k$ for the source problem. Parameterized reductions are allowed to take time that is upper bounded by a function of the parameter $k$ and a polynomial in the input size.

\subsection{Previous results}

By the celebrated Courcelle's theorem [8], all graph properties definable in Monadic Second Order (MSO) logic can be decided in linear time on graphs of bounded treewidth. For every fixed $r$, deciding whether a graph is $r$-colorable is expressible in MSO logic. Since the chromatic number of a graph is at most its treewidth plus one, on graphs of treewidth $t$, the GRAPH Coloring problem is solvable in time $O(f(t) \cdot n)$. Thus GRAPH CoLORING is FPT parameterized by the treewidth of the input graph.

Many NP-complete variations of GRAPH COLORING are solvable in polynomial time on graphs of constant treewidth $t$, however the running time of all these algorithms is $n^{O(t)}$. For example, Jansen and Scheffler described a dynamic programming algorithm for the LIST COLORING problem that runs in time $O\left(n^{t+2}\right)$ [20]. PRECOLORING EXTENSION can also be solved in time $O\left(n^{t+2}\right)$ for graphs of treewidth at most $t$ [20]. The EQUITABLE CoLORING problem is also known to be solvable in time $n^{O(t)}$ [4].

\subsection{Our results}

We give a proof that the running time $n^{O(t)}$ of algorithms from $[4,20]$ for different variants of GRAPH CoLORING is essentially the best we can hope for up to a widely believed assumption $F P T \neq W[1]$. In particular, we show that

- List Coloring, Precoloring Extension, and Equitable Coloring are W[1]-hard for parameter $t$.

To the best of our knowledge, these are the first nontrivial results on the hardness of parameterization by the treewidth of a graph, apart from the NP-hardness of some problems (such as BANDWIDTH) on trees.

While our complexity results can create an impression that any interesting extension of GRAPH CoLORING is W[1]-hard, when parameterized by the treewidth, fortunately, this is not the case. In particular, we show that the LIST CHROMATIC NUMBER problem (known to be $\Pi_{2}^{p}$-complete for any fixed $r \geq 3$ [18]) can be computed in linear time for any fixed treewidth bound $t$. This shows the diversity of coloring problems when parameterized by treewidth.

\section{Some coloring problems that are hard for treewidth}

We tend to think that "all" (or almost all) combinatorial problems are easy for bounded treewidth, but in the case of structured coloring problems, the game is more varied in outcome.

\subsection{LIST COLORING and PReColoRING EXTENSION are W[1]-hard parameterized by treewidth}

There is a simple reduction to the List Coloring (when parameterized by the treewidth $t$ ) from the Multicolor CLIQUE problem which is defined as follows:

Multicolor CliQue : The problem takes as input a graph $G$ together with a proper $k$-coloring of the vertices of $G$. The question is whether there is a $k$-clique in $G$ consisting of exactly one vertex of each color.

The Multicolor CliQue problem is known to be $W[1]$-complete [15] (by a simple reduction from the ordinary CliQue). Starting a reduction from colored versions of different problems has many advantages and gives us a schematic way to design gadgets. Let $V[i]$ be the set of vertices in the color class $i$ and $E[i, j]$ be the set of edges between color class $i$ and $j$. Then we can assume that $|V[i]|=N$ for all $i$, and that $|E[i, j]|=M$ for all $i<j$, that is, we can assume that the vertex color classes of $G$, and also the edge sets between them, have uniform sizes. For a simple justification of this assumption consider the following. We reduce MulTiCOLOR CLIQUe to itself. Let $\mathcal{S}_{k}$ be the set of permutation of $\{1, \ldots, k\}$. Given a $k$ colored graph $G$ and a permutation $\sigma \in \mathcal{S}_{k}$ by $G_{\sigma}$ we mean the graph $G$ where the color class $i$ is colored with $\sigma(i)$. Now given a $k$ colored graph $G$ of Multicolor CliQue we take $G^{\prime}$ a union of $k$ ! disjoint copies of $G$, one for each permutation of the color set. That is, $G^{\prime}=\bigcup_{\sigma \in \mathcal{S}_{k}} G_{\sigma}$. Clearly $G^{\prime}$ has the property that every color class is of same size and between every pair of color class we have the same set of edges. Furthermore $G$ has a multicolored clique of size $k$ if and only if $G^{\prime}$ has.

Now we show that the LIST COLORING problem on graphs of treewidth $t$ is W[1]-hard when parameterized by treewidth. Given the source instance $G$ of MULTICOLOR CLIQUE problem, we construct an instance $G^{\prime}$ of LIST Coloring that admits a proper choice of color from each list if and only if the source instance $G$ has a multicolor $k$-clique. The colors on the lists of vertices in $G^{\prime}$ are in one-to-one correspondence with the vertices of $G$. For simplicity of arguments we do not distinguish between a vertex $v$ of $G$ and the color $v$ which appears in the list assigned to the vertices of $G^{\prime}$. The instance $G^{\prime}$ is constructed as follows: 


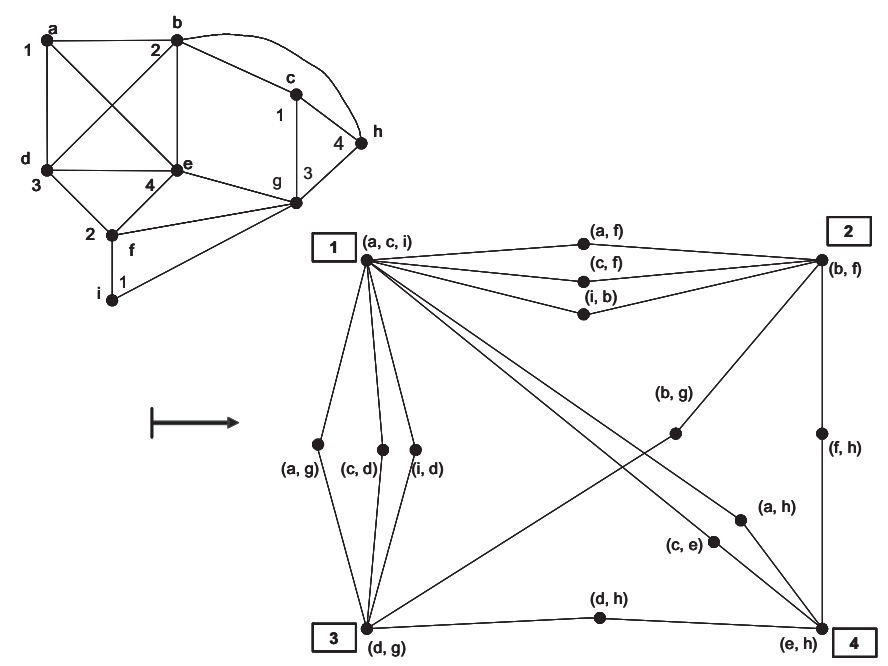

Fig. 1. Example of the reduction from Multicolor CliQue to List Coloring.

1. There are $k$ vertices $v[i]$ in $G^{\prime}, i=1, \ldots, k$, one for each color class of $G$, and the list assigned to $v[i]$ consists of the colors corresponding to the vertices in $G$ of color $i$ that is $L_{v[i]}=\{V[i]\}$.

2. For $i \neq j$, there is a degree two vertex in $G^{\prime}$ adjacent to $v[i]$ and $v[j]$ for each pair $x, y$ of nonadjacent vertices in $G$, where $x$ has color $i$ and $y$ has color $j$. This vertex is labeled $v_{i, j}[x, y]$ and has $\{x, y\}$ as its list.

This completes the construction. As example of the reduction is shown in Fig. 1. The figure shows an example for the parameter value $k=4$.

The treewidth of $G^{\prime}$ is bounded by $k$ as the graph obtained after removing the vertices $v[i], 1 \leq i \leq k$, from $G^{\prime}$ is an empty graph and hence has treewidth 0 (anyway it is well known that degree 2 vertices do not increase the treewidth of a graph). Now, if $G$ has a multicolor clique $K$ then we can easily list color $G^{\prime}$. Assign $v[i]$ with the vertex (color in $G^{\prime}$ ) corresponding to the color class $V[i]$ in the multicolor clique $K$. Now it is easy to see that every degree 2 vertex in $G^{\prime}$ has at least one color free in its list, as the pair of colors in the list correspond to nonadjacent vertices in $G$. For the other direction, we show that the vertices of $G$, corresponding to the colors assigned to $v[i]$ 's in a list coloring of $G^{\prime}$, forms a clique. This follows since two vertices $u$ and $v$ of $G$ belonging to different color classes do not appear together on a list of some degree 2 vertices in $G^{\prime}$ if and only if they have an edge $u v$ between them in $G$. This results in the following theorem.

Theorem 1. LIST COLORING parameterized by treewidth is W[1]-hard.

To show that Precoloring EXTENSION is also W[1]-hard when parameterized by treewidth, we reduce from the LisT Coloring problem, by simply using many precolored vertices of degree 1 to enforce the lists. This construction does not increase the treewidth. More precisely given an instance of $G=(V, E)$ of List CoLoRING we construct an instance $G^{\prime}$ of Precoloring Extension as follows. Let $\mathcal{C}=\bigcup_{v \in V} L_{v}$. Now for every vertex $v \in V$, we add $\ell=|\mathcal{C}| \backslash\left|L_{v}\right|$ vertices of degree 1 and make them adjacent to $v$. Let the set of newly added degree 1 vertices that are adjacent to $v$ be called $S(v)$. We color the vertices of $S(v)$ with colors in $\mathcal{C} \backslash L_{v}$ where each color is used exactly once. This completes the description of $G^{\prime}$, an instance of Precoloring EXTEnsion. Thus we have:

Theorem 2. PReCOLORING EXTENSION parameterized by treewidth is W[1]-hard.

\subsection{EquitABLE Coloring is W[1]-hard parameterized by treewidth}

The EQuitabLE Coloring problem is a classical problem with a long history starting from 1960s [19,25]. Bodlaender and Fomin have shown that determining whether a graph of treewidth at most $t$ admits an equitable coloring, can be solved in time $O\left(n^{O(t)}\right)[4]$.

We consider the parameterized complexity of EQUITABLE CoLORING (ECP) in graphs with bounded treewidth. We actually prove a stronger result than the one we have so far stated. We show that when ECP is parameterized by $(t, r)$, where $t$ is the treewidth bound, and $r$ is the number of color classes, then the problem is $W[1]$-hard. Before we proceed further we make remarks on what it means to parameterize by two parameters, say $\kappa_{1}$ and $\kappa_{2}$. It essentially means parameterizing by a single parameter $k=\kappa_{1}+\kappa_{2}$. For an example INDEPENDENT SET is known to be $W$ [1] complete parameterized by the solution size $k$ [10], while it is FPT when parameterized by the solution size $k$ and the maximum degree $\Delta$ of the input graph. That is, there is an algorithm for INDEPENDENT SET running in time $O\left(f(k+\Delta) n^{c}\right)$, where $n$ is the input size. We refer to [12, Chapter 1, Section 3] for further examples. A combined parameter or two parameters together sometimes enable a problem to be FPT, while with any one of the parameters they are $W[1]$ hard. For an example INDEPENDENT SET is 
not known to be FPT with either the solution size as the parameter or the maximum degree of the input graph. However, when we show that a problem is $W[i]$-hard, $i \geq 1$, with a combined parameter then it also means that the problem is $W[i]$-hard with any one parameter of the combination. Next we show that ECP is $W[1]$-hard parameterized by $(t, r)$ or $t+r$.

In this section, we show a reduction based on a methodology which is sometimes termed as edge representation strategy for the parameterized reduction from MulTiColor CLIQUE. This strategy is very basic and is useful for many reductions. Note that the instance $G=(V, E)$ of Multicolor Clique has its vertices colored by the integers $1, \ldots, k$. Let $V[i]$ denote the set of vertices of color $i$, and let $E[i, j]$, for $1 \leq i<j \leq k$, denote the set of edges $e=u v$, where $u \in V[i]$ and $v \in V[j]$. We also assume that $|V[i]|=N$ for all $i$, and that $|E[i, j]|=M$ for all $i<j$, that is, the vertex color classes of $G$, and also the edge sets between them, have uniform sizes.

In what follows next we adhere to edge representation strategy and form gadgets in the context of reduction from Multicolor Clique to Equitable Coloring Problem on graphs with bounded treewidth. To show the desired reduction, we introduce two intermediate problems.

List Equitable Coloring Problem (LECP): Given an input graph $G=(V, E)$, lists $L_{v}$ of colors for every vertex $v \in V$ and a positive integer $r$; does there exist a proper coloring $f$ of $G$ with exactly $r$ colors that for every vertex $v \in V$ uses a color from its list $L_{v}$ such that for any two color class, $V_{i}$ and $V_{j}$ of the coloring $f,|| V_{i}|-| V_{j}|| \leq 1$ ?

Number List Coloring Problem (NLCP): Given an input graph $G=(V, E)$, lists $L_{v}$ of colors for every vertex $v \in V$, a function $h: \cup_{v \in V} L_{v} \rightarrow \mathbb{N}$, associating a number to each color, and a positive integer $r$; does there exist a proper coloring $f$ of $G$ with $r$ colors that for every vertex $v \in V$ uses a color from its list $L_{v}$, such that any color class $V_{c}$ of the coloring $f$ is of size $h(c)$ ?

List analogues of equitable coloring have been previously studied by Kostochka et al. [22]. Our main effort is in the reduction of the MULTICOLOR CLIQUE problem to NLCP.

We will use the following sets of colors in our construction of an instance of NLCP:

1. $\mathcal{S}=\{\sigma[i, j]: 1 \leq i \neq j \leq k\}$

2. $\mathcal{S}^{\prime}=\left\{\sigma^{\prime}[i, j]: 1 \leq i \neq j \leq k\right\}$

3. $\mathcal{T}=\left\{\tau_{i}[r, s]: 1 \leq i \leq k, 1 \leq r<s \leq k, r \neq i, s \neq i\right\}$

4. $\mathcal{T}^{\prime}=\left\{\tau_{i}^{\prime}[r, s]: 1 \leq i \leq k, 1 \leq r<s \leq k, r \neq i, s \neq i\right\}$

5. $\mathcal{E}=\{\epsilon[i, j]: 1 \leq i<j \leq k\}$

6. $\mathcal{E}^{\prime}=\left\{\epsilon^{\prime}[i, j]: 1 \leq i<j \leq k\right\}$.

Note that $|\mathcal{S}|=\left|\mathcal{S}^{\prime}\right|=2\left(\begin{array}{l}k \\ 2\end{array}\right)$, that is, there are distinct colors $\sigma[2,3]$ and $\sigma[3,2]$, etc. In contrast, the colors $\tau_{i}[r, s]$ are only defined for $r<s$.

We associate with each vertex and edge of $G$ a pair of (unique) identification numbers. The up-identification number $v$ [up] for a vertex $v$ should be in the range $\left[n^{2}+1, n^{2}+n\right]$, if $G$ has $n$ vertices and it could be chosen arbitrarily, but uniquely. Similarly, the up-identification number [up] of an edge $e$ of $G$ can be assigned (arbitrarily, but uniquely) in the range $\left[2 n^{2}+1,2 n^{2}+m\right]$, assuming $G$ has $m$ edges.

Choose a suitably large positive integer $Z_{0}$, for example $Z_{0}=n^{3}$, and define the down-identification number $v$ [down] for a vertex $v$ to be $Z_{0}-v[u p]$, and similarly for the edges $e$ of $G$, define the down-identification number $e\left[\right.$ down] to be $Z_{0}-e[u p]$.

Choose a second large positive integer, $Z_{1}>>Z_{0}$, for example, we may take $Z_{1}=n^{6}$.

Next we describe various gadgets and the way they are combined in the reduction. First we describe the gadget which encodes the selection of the edge going between two particular color classes in $G$. In other words, we will think of the representation of a $k$-clique in $G$ as involving the selection of edges (with each edge selected twice, once in each direction) between the color classes of vertices in $G$, with gadgets for selection, and to check two things: (1) that the selections in opposite color directions match, and (2) that the edges chosen from color class $V[i]$ going to $V[j]$ (for various $j \neq i$ ) all emanate from the same vertex in $V[i]$.

There are $2\left(\begin{array}{l}k \\ 2\end{array}\right)$ groups of gadgets, one for each pair of color indices $i \neq j$. If $1 \leq i<j \leq k$, then we will refer to the gadgets in the group $\mathcal{G}[i, j]$ as forward gadgets, and we will refer to the gadgets in the group $\mathcal{G}[j, i]$ as backward gadgets.

If $e \in E[i, j]$, then there is one forward gadget corresponding to $e$ in the group $\mathcal{G}[i, j]$, and one backward gadget corresponding to $e$ in the group $\mathcal{G}[j, i]$. The construction of these gadgets is described as follows:

\subsubsection{The forward gadget corresponding to $e=u v \in E[i, j]$}

The gadget has a root vertex $r[i, j, e]$, and consists of a tree of height 2 . The list assigned to this root vertex contains two colors: $\sigma[i, j]$ and $\sigma^{\prime}[i, j]$. The root vertex has $Z_{1}+1$ children, and each of these is also assigned the two-element list containing the colors $\sigma[i, j]$ and $\sigma^{\prime}[i, j]$. One of the children vertices is distinguished, and has $2 k$ groups of further children: 
- $e[u p]$ children assigned the list $\left\{\sigma^{\prime}[i, j], \epsilon[i, j]\right\}$.

- $e[$ down $]$ children assigned the list $\left\{\sigma^{\prime}[i, j], \epsilon^{\prime}[i, j]\right\}$.

- For each $r$ in the range $j<r \leq k, u[u p]$ children assigned the list $\left\{\sigma^{\prime}[i, j], \tau_{i}[j, r]\right\}$.

- For each $r$ in the range $j<r \leq k, u[$ down $]$ children assigned $\left\{\sigma^{\prime}[i, j], \tau_{i}^{\prime}[j, r]\right\}$.

- For each $r$ in the range $1 \leq r<j, u[$ down $]$ children assigned $\left\{\sigma^{\prime}[i, j], \tau_{i}[r, j]\right\}$.

- For each $r$ in the range $1 \leq r<j, u[u p]$ children assigned the list $\left\{\sigma^{\prime}[i, j], \tau_{i}^{\prime}[r, j]\right\}$.

Thus the number of grandchildren of $r[i, j, e]$ is

$$
\begin{aligned}
& e[\text { up }]+e[\text { down }]+(k-j) u[u p]+(k-j) u[\text { down }]+(j-1) u[\text { down }]+(j-1) u[u p] \\
& \quad=Z_{0}+(k-j) Z_{0}+(j-1) Z_{0} \\
& \quad=k Z_{0} .
\end{aligned}
$$

2.2.2. The backward gadget corresponding to $e=u v \in E[i, j]$

The gadget has a root vertex $r[j, i, e]$, and consists of a tree of height 2 . The list assigned to this root vertex contains two colors: $\sigma[j, i]$ and $\sigma^{\prime}[j, i]$. The root vertex has $Z_{1}+1$ children, and each of these is also assigned the two-element list containing the colors $\sigma[j, i]$ and $\sigma^{\prime}[j, i]$. One of the children vertices is distinguished, and has $2 k$ groups of further children:

- $e[u p]$ children assigned the list $\left\{\sigma^{\prime}[j, i], \epsilon^{\prime}[i, j]\right\}$.

- $e[$ down $]$ children assigned the list $\left\{\sigma^{\prime}[j, i], \epsilon[i, j]\right\}$.

- For each $r$ in the range $i<r \leq k, v[u p]$ children assigned the list $\left\{\sigma^{\prime}[j, i], \tau_{j}[i, r]\right\}$.

- For each $r$ in the range $i<r \leq k, v\left[\right.$ down] children assigned $\left\{\sigma^{\prime}[j, i], \tau_{j}^{\prime}[i, r]\right\}$.

- For each $r$ in the range $1 \leq r<i, v\left[\right.$ down] children assigned $\left\{\sigma^{\prime}[j, i], \tau_{j}[r, i]\right\}$.

- For each $r$ in the range $1 \leq r<i, v[u p]$ children assigned the list $\left\{\sigma^{\prime}[j, i], \tau_{j}^{\prime}[r, i]\right\}$.

Thus the number of grandchildren of $r[j, i, e]$ is

$$
\begin{aligned}
& e[\text { up }]+e[\text { down }]+(k-i) v[\text { up }]+(k-i) v[\text { down }]+(i-1) v[\text { down }]+(i-1) v[\text { up }] \\
& \quad=Z_{0}+(k-i) Z_{0}+(i-1) Z_{0} \\
& \quad=k Z_{0} .
\end{aligned}
$$

2.2.3. The numerical targets (function $h$ )

1. For all $c \in\left(\mathcal{T} \cup \mathcal{T}^{\prime}\right), h(c)=Z_{0}$.

2. For all $c \in\left(\mathcal{E} \cup \mathcal{E}^{\prime}\right), h(c)=Z_{0}$.

3. For all $c \in \mathcal{S}, h(c)=(M-1)\left(Z_{1}+1\right)+1$.

4. For all $c \in \mathcal{S}^{\prime}, h(c)=(M-1)+\left(Z_{1}+1\right)+k(M-1) Z_{0}$.

Given the source instance $G$ of Multicolor CLIQue problem, we construct an instance $G^{\prime}$ of NLCP that admits a proper choice of color from each list as well as that each color $c$ appears on exactly $h(c)$ vertices (that is, meets its numerical requirement) if and only if the source instance $G$ has a multicolor $k$-clique. The instance $G^{\prime}$ is essentially the disjoint union of the edge gadgets. That is,

$$
G^{\prime}=\bigcup_{1 \leq i \neq j \leq k} \mathcal{G}[i, j]
$$

The $h$ function is defined as above. That completes the formal description of the reduction from MULTICOLOR CLIQUE to NLCP. We turn now to some motivating remarks about the design of the reduction.

2.2.4. Remarks on the colors, their numerical targets, and their role in the reduction

(1) There are $2\left(\begin{array}{l}k \\ 2\end{array}\right)$ groups of gadgets. Each edge of $G$ gives rise to two gadgets. Between any two color classes of $G$ there are precisely $M$ edges, and therefore $M \cdot\left(\begin{array}{l}k \\ 2\end{array}\right)$ edges in $G$ in total. Each group of gadgets therefore contains $M$ gadgets. The gadgets in each group have two "helper" colors. For example, the group of gadgets $\mathcal{G}[4,2]$ has the helper colors $\sigma[4,2]$ and $\sigma^{\prime}[4,2]$. The role of the gadgets in this group is to indicate a choice of an edge going from a vertex in the color class $V[4]$ of $G$ to a vertex in the color class $V$ [2] of $G$. The role of the $2\left(\begin{array}{l}k \\ 2\end{array}\right)$ groups of gadgets is to represent the selection of $\left(\begin{array}{l}k \\ 2\end{array}\right)$ edges of $G$ that form a $k$-clique, with each edge chosen twice, once in each direction. If $i<j$ then the choice is represented by the coloring of the gadgets in the group $\mathcal{G}[i, j]$, and these are the forward gadgets of the edge choice. If $j<i$, then the gadgets in $\mathcal{G}[i, j]$ are backward gadgets (representing the edge selection in the opposite direction, relative to the ordering of the color classes of $G$ ). The numerical targets for the colors in $\mathcal{S} \cup \mathcal{S}^{\prime}$ are chosen to force exactly one 
edge to be selected (forward or backward) by each group of gadgets, and to force the gadgets that are colored in a way that indicates the edge was not selected into being colored in a particular way (else the numerical targets cannot be attained). The numerical targets for these colors are complicated, because of this role (which is asymmetric between the pair of colors $\sigma[i, j]$ and $\left.\sigma^{\prime}[i, j]\right)$.

(2) The colors in $\mathcal{T} \cup \mathcal{T}^{\prime}$ and $\mathcal{E} \cup \mathcal{E}^{\prime}$ are organized in symmetric pairs, and each pair is used to transmit (and check) information. Due to the enforcements alluded to above, each "selection" coloring of a gadget (there will be only one possible in each group of gadgets) will force some number of vertices to be colored with these pairs of colors, which can be thought of as an information transmission. For example, when a gadget in $\mathcal{G}[4,2]$ is colored with a "selection" coloring, this indicates that the edge from which the gadget arises is selected as the edge from the color class $V[4]$ of $G$, to the color class $V[2]$. There is a pair of colors that handles the information transmission concerning which edge is selected between the groups $\mathcal{G}[2,4]$ and $\mathcal{G}[4,2]$. (Of course, something has to check that the edge selected in one direction, is the same as the edge selected in the other direction.) There is something elegant about the dual-color transmission channel for this information. Each vertex and edge has two unique identification numbers, "up" and "down", that sum to $Z_{0}$. To continue the concrete example, $\mathcal{G}[4,2]$ uses the (number of vertices colored by the) pair of colors $\epsilon[2,4]$ and $\epsilon^{\prime}[2,4]$ to communicate to $\mathcal{G}[2,4]$ about the edge selected. The signal from one side consists of $e[u p]$ vertices colored $\epsilon[2,4]$ and $e$ [down] vertices colored $\epsilon^{\prime}[2,4]$. The signal from the other side consists of $e$ [down] vertices colored $\epsilon[2,4]$ and $e[u p]$ vertices colored $\epsilon^{\prime}[2,4]$. Thus the numerical targets for these colors allow us to check whether the same edge has been selected in each direction (if each color target of $Z_{0}$ is met). There is the additional advantage that the amount of signal in each direction is the same: in each direction a total of $Z_{0}$ colored vertices, with the two paired colors, constitutes the signal. This means that, modulo the discussion in (1) above, when an edge is not selected, the corresponding nonselection coloring involves uniformly the same number (i.e., $\left.Z_{0}\right)$ of vertices colored "otherwise" for each of the $(M-1)$ gadgets colored in the non-selection way: this explains (part of) the $k(M-1) Z_{0}$ term in (4) of the numerical targets.

(3) In a similar manner to the communication task discussed above, each of the $k-1$ groups of gadgets $\mathcal{G}\left[i\right.$, $\left._{-}\right]$need to check that each has selected an edge from $V[i]$ that originates at the same vertex in $V[i]$. Hence there are pairs of colors that provide a communication channel similar to that in (2) for this information. This role is played by the colors in $\mathcal{T} \cup \mathcal{T}^{\prime}$. (Because of the bookkeeping issues, this becomes somewhat intricate in the formal definition of the reduction.)

The above remarks are intended to aid an intuitive understanding of the reduction. We now return to a more formal argument.

Claim 1. If $G$ has a k-multicolor clique, then $G^{\prime}$ is a yes-instance to NLCP.

Proof. The proof of this claim is relatively straightforward. The gadgets corresponding to the edges of a $k$-clique in $G$ are colored in a manner that indicates "selected" (for both the forward and the backward gadgets) and all other gadgets are colored in manner that indicates "non-selected". The coloring that corresponds to "selected" colors the root vertex with the color $\sigma[i, j]$, and this forces the rest of the coloring of the gadget. The coloring that corresponds to "non-selected" colors the root vertex with the color $\sigma^{\prime}[i, j]$. In this case the coloring of the rest of the gadget is not entirely forced, but if the grandchildren vertices of the gadget are also colored with $\sigma^{\prime}[i, j]$, then all the numerical targets will be met. We substantiate below how the numerical targets are met.

A color $\sigma[i, j] \in \mathcal{S}$ is used only on the gadgets belonging to $\mathcal{G}[i, j]$. There are $M$ edge gadgets in $\mathcal{G}[i, j]$ and $\sigma[i, j]$ is used once on the root of a gadget (the one which indicates selection) and on the remaining $M-1$ gadgets it is used on children of roots. This implies that $\sigma[i, j]$ is used on $1+(M-1)\left(Z_{1}+1\right)$ vertices, meeting its required numerical target. Similarly we know that $\sigma^{\prime}[i, j] \in \mathcal{S}^{\prime}$ is used only on the gadgets belonging to $\mathcal{G}[i, j]$. This is used on the roots of $M-1$ edges gadgets and on the grandchildren of these gadgets. For the remaining one gadget it is used on the children of root. This implies that it is used on $(M-1)+(M-1) k Z_{0}+\left(Z_{1}+1\right)$ vertices and hence meets its numerical target. Now we argue about colors in $\mathcal{T}$ and $\mathcal{T}^{\prime}$. A color $\tau_{i}[r, s], r<s$, is used only on the gadgets in $\mathcal{G}[i, r]$ and $\mathcal{G}[i, s]$ and appears only on the list of grandchildren. We only use this color on a gadget when the root is colored with $\sigma[i, r]$ (that is the gadget corresponds to selection) among the gadgets of $\mathcal{G}[i, r]$ and $\sigma[i, s]$ among the gadgets of $\mathcal{G}[i, s]$. Hence if $v$ is the vertex in the clique from the color class $i$ then the color $\tau_{i}[r, s]$ is used $v[u p]$ times on the vertices of gadgets of $\mathcal{G}[i, r]$ and is used $v$ [down] times on the vertices of gadgets of $\mathcal{G}[i, r]$. This shows that $\tau_{i}[r, s]$ is used exactly $Z_{0}$ times on the vertices and hence meets its numerical target. One can similarly show for a color $\tau_{i}^{\prime}[r, s]$. Finally we argue that colors in $\mathcal{E}$ and $\mathcal{E}^{\prime}$ meet their numerical target. A color $\epsilon[i, j]$ is used only among the gadgets of $\mathcal{G}[i, j]$ and $\mathcal{G}[j, i]$ and again we use this color only on the vertices of gadgets where the roots are colored with $\sigma[i, j]$ and $\sigma[j, i]$, respectively. Hence if $e$ is the edge selected in the clique between color class $i$ and color class $j$ then if $i<j$ the color $\epsilon[i, j]$ is used $e[u p]$ times on the vertices of gadgets of $\mathcal{G}[i, j]$ and $e$ [down] times on the vertices of gadgets of $\mathcal{G}[i, j]$, else vice-versa. Either way it is used $e[u p]+e[$ down $]=Z_{0}$ times. One can similarly show that a color $\epsilon^{\prime}[i, j]$ meets its numerical target.

Claim 2. Suppose that $\Gamma$ is a list coloring of $G^{\prime}$ that meets all the numerical targets. Then in each group of gadgets, exactly one gadget is colored in a way that indicates "selection". That is, among the gadgets of $\mathcal{G}[i, j]$ exactly one of the gadget's root is colored with $\sigma[i, j]$. 
Proof. We argue this as follows. We first show that there cannot be two gadgets in any group $\mathcal{G}[i, j]$ such that its roots are colored with $\sigma[i, j]$. If this happens then the color $\sigma^{\prime}[i, j]$ has to be used on the children of these roots. This implies that $\sigma^{\prime}[i, j]$ will at least be used on $2 Z_{1}+2 \geq 2 n^{6}+2$ vertices. However the number of times the color $\sigma^{\prime}[i, j]$ has to be used on vertices is

$$
\begin{aligned}
(M-1)+\left(Z_{1}+1\right)+k(M-1) Z_{0} & \leq \frac{n(n-1)}{2}+n^{6}+n\left(\frac{n(n-1)}{2}-1\right) n^{3} \\
& \leq \frac{n^{2}}{2}-\frac{n}{2}+n^{6}+\frac{n^{6}}{2}-\frac{n^{5}}{2}-n^{4} \\
& <2 n^{6},
\end{aligned}
$$

which is less than what we need to use when we color roots of two gadgets in the group $\mathcal{G}[i, j]$ with $\sigma[i, j]$. Now we show that if there exists no gadget in the group $\mathcal{G}[i, j]$ such that its roots is colored with $\sigma[i, j]$ then also the numerical targets for the colors in $\mathcal{S} \cup \mathcal{S}^{\prime}$ are not met. In this case we know that all the roots of the gadgets in $\mathcal{G}[i, j]$ are colored with $\sigma^{\prime}[i, j]$. Hence all the children of the roots of the gadgets in $\mathcal{G}[i, j]$ are colored with $\sigma[i, j]$. This implies that $\sigma[i, j]$ will be used on $M\left(Z_{1}+1\right)=M Z_{1}+M$ vertices. However the number of times the color $\sigma[i, j]$ has to be used on vertices is

$$
\begin{aligned}
(M-1)\left(Z_{1}+1\right)+1 & =M Z_{1}+M-Z_{1}-1+1 \\
& <M Z_{1}+M
\end{aligned}
$$

as $Z_{1}>M$. This is less than what we need to use when we color root of no gadget in the group $\mathcal{G}[i, j]$ with $\sigma[i, j]$. This implies that among the gadgets of $\mathcal{G}[i, j]$ exactly one of the gadget's root is colored with $\sigma[i, j]$. This concludes the proof.

Claim 3. Suppose that $\Gamma$ is a list coloring of $G^{\prime}$ that meets all the numerical targets. Then in each group of gadgets, every gadget that is not colored in a way that indicates "selection" must have all of its grandchildren vertices colored with the appropriate color in $\mathcal{S}^{\prime}$. That is, all the gadgets of $\mathcal{G}[i, j]$ where the root is colored with $\sigma^{\prime}[i, j]$, all its grandchildren are also colored with $\sigma^{\prime}[i, j]$.

Proof. Suppose to the contrary that there exists a group $\mathcal{G}[i, j]$ such that it has a gadget $\mathcal{L}$ such that its root is colored with $\sigma^{\prime}[i, j]$, and at least one grandchildren is not colored with $\sigma^{\prime}[i, j]$. By Claim 2 , we know that there exists exactly one gadget, say $\mathcal{L}^{\prime} \neq \mathcal{L}$ in $\mathcal{G}[i, j]$ whose root is colored with $\sigma[i, j]$. All the children of the root of $\mathcal{L}^{\prime}$ is colored with $\sigma^{\prime}[i, j]$ and hence no grandchildren of the root of $\mathcal{L}^{\prime}$ is colored with $\sigma^{\prime}[i, j]$. This implies that $\sigma^{\prime}[i, j]$ only appears on the roots of $M-1$ gadgets of $\mathcal{G}[i, j]$ and their grandchildren. Hence the vertices on which $\sigma^{\prime}[i, j]$ is used are (a) $(M-1)$ roots; (b) $Z_{1}+1$ children of the root of $\mathcal{L}^{\prime}$; and $(\mathrm{c})$ at most $(M-2) k Z_{0}+\left(k Z_{0}-1\right)$ on the grandchildren of roots of gadgets in $\mathcal{G}[i, j]$. The last assertion follows from our assumption that at least one grandchildren of the root of $\mathcal{L}$ is not colored with $\sigma^{\prime}[i, j]$. This implies that the number of times $\sigma^{\prime}[i, j]$ is used is bounded above by $(M-1)+\left(Z_{1}+1\right)+\left((M-1) k Z_{0}\right)-1$ and hence $\sigma^{\prime}[i, j]$ does not meet its numerical target. This proves that all the gadgets of $\mathcal{G}[i, j]$ where the root is colored with $\sigma^{\prime}[i, j]$, its all grandchildren are also colored with $\sigma^{\prime}[i, j]$.

Claim 4. Suppose that $\Gamma$ is a list coloring of $G^{\prime}$ that meets all the numerical targets. Then if $r[i, j, e]$ is colored with $\sigma[i, j]$ then $r[j, i, e]$ is colored with $\sigma[j, i]$.

Proof. Without loss of generality assume that $i<j$. If $r[i, j, e]$ is colored with $\sigma[i, j]$ then by Claims 2 and 3 we know that this is the unique gadget of $\mathcal{G}[i, j]$ such that the grandchildren of $r[i, j, e]$ are not colored with $\sigma^{\prime}[i, j]$. Hence $e[u p]$ grandchildren of $r[i, j, e]$ are colored with $\epsilon[i, j]$ and $e[$ down $]$ grandchildren of $r[i, j, e]$ are colored with $\epsilon^{\prime}[i, j]$. But to meet the numerical targets of $\epsilon[i, j]$ and $\epsilon^{\prime}[i, j]$ we need $Z_{0}-e[u p]$ and $Z_{0}-e[$ down $]$ more vertices, respectively, to be colored with $\epsilon[i, j]$ and $\epsilon^{\prime}[i, j]$. However, $\epsilon[i, j]$ and $\epsilon^{\prime}[i, j]$ appear $e[$ down $]$ and $e[u p]$ times, respectively, only on the grandchildren of $r[j, i, e]$. This together with Claims 2 and 3 imply that $r[j, i, e]$ is the root of the gadget in $\mathcal{G}[j, i]$ that is colored with $\sigma[j, i]$.

Claim 5. Suppose that $\Gamma$ is a list coloring of $G^{\prime}$ that meets all the numerical targets. Fix an $1 \leq i \leq k$, then all the roots $r[i, j, e]$, $j \neq i$, that are colored with $\sigma[i, j]$ has the property that all the edges e that appear in the definition of $r[i, j, e]$ are incident to the same vertex in the color class $i$ of the graph $G$.

Proof. We prove it by contradiction. Suppose we have two roots $r\left[i, j, e_{1}\right]$ and $r\left[i, \ell, e_{2}\right]$ that are colored with $\sigma[i, j]$ and $\sigma[i, \ell]$, respectively, and $i \neq j, i \neq \ell$ and $j \neq \ell$. Here $e_{1}=u_{1} v_{1}$ and $e_{2}=u_{2} v_{2}$ are edges between color class $i$ and $j$ and between color class $i$ and $\ell$, respectively. Furthermore $u_{1} \neq u_{2}$ and both are colored with $i$ in $G$. Without loss of generality also assume that $i<j<\ell$. Now we look at the number of times the colors $\tau_{i}[j, \ell]$ and $\tau_{i}^{\prime}[j, \ell]$ are used in the gadget whose root is $r\left[i, j, e_{1}\right]$. By Claims 2 and 3 we know that this is the unique gadget of $\mathcal{G}[i, j]$ such that the grandchildren of $r\left[i, j, e_{1}\right]$ are not colored with $\sigma^{\prime}[i, j]$ but with other colors. Thus $\tau_{i}[j, \ell]$ is used on $u_{1}[u p]$ vertices and $\tau_{i}^{\prime}[j, \ell]$ is used on $u_{1}[$ down] 
vertices of the gadget whose root is $r\left[i, j, e_{1}\right]$. Now to meet the numerical requirements of $\tau_{i}[j, \ell]$ and $\tau_{i}^{\prime}[j, \ell]$ we need to color more vertices. However these colors can only be given to the vertices of the gadget whose root is $r\left[i, \ell, e_{2}\right]$ and they need to meet their numerical requirements by coloring the appropriate number of vertices in this gadget. Thus we know that the number of vertices that are assigned the color $\tau_{i}[j, \ell]$ and $\tau_{i}^{\prime}[j, \ell]$ among the vertices of the gadget rooted at $r\left[i, \ell, e_{2}\right]$ are $u_{2}$ [down] and $u_{2}$ [up], respectively. Thus the number of times we use $\tau_{i}[j, \ell]$ is $u_{1}[u p]+u_{2}[$ down] and the number of times we use $\tau_{j}^{\prime}[j, \ell]$ is $u_{1}[$ down $]+u_{2}[u p]$. But $u_{1}[u p]+u_{2}[$ down $] \neq Z_{0}$ and $u_{1}[$ down $]+u_{2}[u p] \neq Z_{0}$. The last assertion follows since given an up identification number there is an unique down identification number to make it equal to $Z_{0}$. Hence $u_{1}$ must be equal to $u_{2}$, a contradiction to our assumption. Thus all the roots $r[i, j, e], j \neq i$, that are colored with $\sigma[i, j]$ has the property that all the edges $e$ that appear in the definition of $r[i, j, e]$ are incident to the same vertex in the color class $i$ of the graph $G$.

Finally we have the following claim.

Claim 6. Suppose that $\Gamma$ is a list coloring of $G^{\prime}$ that meets all the numerical targets. Then $G$ has a multicolor clique of size $k$.

Proof. Let $F$ be the set of edges that appears in the gadget whose root $r[i, j, e]$ is colored with $\sigma[i, j]$ by $\Gamma$. First by Claim 2 we know that for every $i \neq j$ there is exactly one gadget in $\mathcal{G}[i, j]$ whose root is colored with $\sigma[i, j]$. By Claim 4 we know that if $e$ appears in $r[i, j, e]$ then $e$ also appears in $r[j, i, e]$. Furthermore by Claim 5 we know that all the edges selected in $F$ whose end-points are colored with $i$ are same. That is, this process only selects a vertex from a color class $i$ and all the edges emanate from the same vertex. All this shows that the edges in $F$ form a clique in $G$.

Now using Claims 1 and 6 we obtain the following.

Theorem 3. NLCP is W[1]-hard for forests, parameterized by the number of colors that appear on the lists.

The reduction from NLCP to LECP is almost trivial, achieved by padding with isolated vertices having single-color lists. The reduction from LECP to ECP is described as follows. We add a clique on $r$ vertices, numbered from 1 to $r$. We connect the vertex $i$ in the clique to all vertices that do not contain $i$ in their list of allowed colors. Clearly, any list coloring of $G$ can be extended to a coloring of $G^{\prime}$ by coloring the vertex $i$ of the clique with color $i$. On the other hand, any coloring of $G^{\prime}$ must color the vertices of the clique with distinct colors. Without loss of generality, the vertex $i$ of the clique is colored with color $i$. Then all neighbors of this vertex, that is, all vertices of $G$ that do not have $i$ in its list, cannot be colored with $i$. Since $G^{\prime}$ is a forest, the treewidth of the resulting graph is at most $r$. This proves the following theorem.

Theorem 4. EQUITABLE COLORING is W[1]-hard, parameterized by treewidth.

\section{List Chromatic Number parameterized by treewidth is FPT}

The notion of the list chromatic number (also known as the choice number) of a graph was introduced by Vizing [30], and independently by Erdös, Rubin and Taylor in 1980 [11]. A celebrated result that gave impetus to the area was proved by Thomassen: every planar graph has list chromatic number at most five [28].

We describe an algorithm for the List CHROMATic NUMBER problem that runs in linear time for any fixed treewidth bound t. Our algorithm employs the machinery of Monadic Second Order logic, due to Courcelle [8] (also [2,6]). At a glance, this may seem surprising, since there is no obvious way to describe the problem in MSO logic - one would seemingly have to quantify over all possible list assignments to the vertices of $G$, and the vocabulary of MSO seems not to provide any way to do this. We employ a "trick" that was first described (to our knowledge) in [5], with further applications described in [7,14].

The essence of the trick is to construct an auxiliary graph that consists of the original input, augmented with additional semantic vertices, so that the whole ensemble has - or can safely be assumed to have - bounded treewidth, and relative to which the problem of interest can be expressed in MSO logic.

A list assignment $L$ with $\left|L_{v}\right| \geq r$ for all $v \in V$ is termed an $r$-list assignment. A list assignment $L$ from which $G$ cannot be properly colored is called bad. Thus, a graph $G$ does not have list chromatic number $\chi_{l}(G) \leq r$, if and only if there is a bad $r$-list assignment for $G$.

The following lemma is crucial to the approach.

Lemma 1. If a graph of treewidth at most $t$ admits any bad $r$-list assignment, then it admits a bad list assignment where the colors are drawn from a set of $(2 t+1) r$ colors.

Proof. First of all, we may note that if $G$ has treewidth bounded by $t$, then $\chi_{l}(G) \leq t+1$ (and similarly, the chromatic number of $G$ is at most $t+1$ ). This follows easily from the inductive definition of $t$-trees. We can therefore assume that $r \leq t+1$.

Fix attention on a width $t$ tree decomposition $\mathcal{D}$ for $G$, where the bags of the decomposition are indexed by the tree $T$. For a node $t$ of $T$, let $\mathcal{D}(t)$ denote the bag associated to the node $t$. Suppose that $L$ is a bad $r$-list assignment for $G$, and let $\mathcal{C}$ 
denote the union of the lists of $L$. For a color $\alpha \in \mathcal{C}$, let $T_{\alpha}$ denote the subforest of $T$ induced by the set of nodes $t$ of $T$ for which $\mathcal{D}(t)$ contains a vertex $v$ of $G$, where the color $\alpha$ occurs in the list $L_{v}$. Let $\mathcal{T}(\alpha)$ denote the set of trees of the forest $T_{\alpha}$. Let $\mathcal{T}$ denote the union of the sets $\mathcal{T}(\alpha)$, taken over all of the colors $\alpha$ that occur in the list assignment $L$ :

$$
\mathcal{T}=\bigcup_{\alpha \in \mathcal{C}} \mathcal{T}(\alpha)
$$

We consider that two trees $T^{\prime}$ and $T^{\prime \prime}$ in $\mathcal{T}$ are adjacent if the distance between $T^{\prime}$ and $T^{\prime \prime}$ in $T$ is at most one. Note that $T^{\prime}$ and $T^{\prime \prime}$ might not be disjoint, so the distance between them can be zero. Let $\mathcal{G}$ denote the graph thus defined: the vertices of $\mathcal{G}$ are the subtrees in $\mathcal{T}$ and the edges are given by the above adjacency relationship.

Suppose that $\mathcal{G}$ can be properly colored by the coloring function $c^{\prime}: \mathcal{T} \rightarrow \mathcal{C}^{\prime}$. We can use such a coloring to describe a modified list assignment $L^{\prime}\left[c^{\prime}\right]$ to the vertices of $G$ in the following way: if $T^{\prime} \in \mathcal{T}(\alpha)$ and $c^{\prime}\left(T^{\prime}\right)=\alpha^{\prime} \in \mathcal{C}^{\prime}$, then replace each occurrence of the color $\alpha$ on the lists $L_{v}$, for all vertices $v$ that belong to bags $\mathcal{D}(t)$, where $t \in T^{\prime}$, with the color $\alpha^{\prime}$.

This specification of $L^{\prime}\left[c^{\prime}\right]$ is consistent, because for any vertex $v$ such that $\alpha \in L_{v}$, there is exactly one tree $T^{\prime} \in \mathcal{T}(\alpha)$ such that $v$ belongs to a bag indexed by nodes of $T^{\prime}$.

Claim 1. If $c^{\prime}$ is a proper coloring of $\mathcal{G}$, and $L$ is a bad list assignment for $G$, then $L^{\prime}\left[c^{\prime}\right]$ is also a bad list assignment for $G$.

This follows because the trees in $\mathcal{G}$ preserve the constraints expressed in having a given color on the lists of adjacent vertices of $G$, while the new colors $\alpha^{\prime}$ can only be used on two different trees $T^{\prime}$ and $T^{\prime \prime}$ when the vertices of $G$ in the bags associated with these trees are at a distance of at least two in $\mathcal{G}$.

Claim 2. The graph $\mathcal{G}$ has treewidth at most $2(t+1) r-1$.

A tree decomposition $\mathcal{D}^{\prime}$ for $\mathcal{G}$ of width at most $2(t+1) r$ can be described as follows. Subdivide each edge $t t^{\prime}$ of $T$ with a node of degree two denoted $s\left(t, t^{\prime}\right)$. Assign to each node $t$ the bag $\mathcal{D}^{\prime}(t)$ consisting of those trees $T^{\prime}$ of $\mathcal{G}$ that include $t$. There are at most $(t+1) r$ such trees. Assign to each node $s\left(t, t^{\prime}\right)$ the bag $\mathcal{D}^{\prime}\left(s\left(t, t^{\prime}\right)\right)=\mathcal{D}^{\prime}(t) \cup \mathcal{D}^{\prime}\left(t^{\prime}\right)$. It is straightforward to verify that this satisfies the requirements of a tree decomposition for $\mathcal{G}$.

The lemma now follows from the fact that $\mathcal{G}$ can be properly colored with $2(t+1) r$ colors.

Theorem 5. The LIST CHROMATIC NuMBER problem, parameterized by the treewidth bound $t$, is fixed-parameter tractable, solvable in linear time for every fixed $t$.

Proof. The algorithm consists of the following steps:

Step 1. Compute in linear time, using Bodlaender's algorithm, a tree-decomposition for $G$ of width at most $t$. Consider the vertices of $G$ to be of type 1 .

Step 2. Introduce $2(t+1) r$ new vertices of type 2, and connect each of these to all vertices of $G$. The treewidth of this augmented graph is at most $t+2(t+1) r=O\left(t^{2}\right)$.

Step 3. The problem can now be expressed in MSO logic. That this is so, is not entirely trivial, and is argued as follows (sketch). We employ a routine extension of MSO logic that provides predicates for the two types of vertices.

If $G$ admits a bad $r$-list assignment, then this is witnessed by a set of edges $F$ between vertices of $G$ (that is, type 1 vertices) and vertices of type 2 (that represent the colors), such that every vertex $v$ of $G$ has degree $r$ relative to $F$. Thus, the $r$ incident $F$-edges represent the colors of $L_{v}$. It is routine to assert the existence of such a set of edges in MSO logic.

The property that such a set of edges $F$ represents a bad list assignment can be expressed as: "For every subset $F^{\prime} \subset F$ such that every vertex of $G$ has degree 1 relative to $F^{\prime}$ (and thus, $F^{\prime}$ represents a choice of a color for each vertex, chosen from its list), there is an adjacent pair of vertices $u$ and $v$ of $G$, such that the represented color choice is the same, i.e., $u$ and $v$ are adjacent by edges of $F^{\prime}$ to the same type 2 (color-representing) vertex." The translation of this statement into formal MSO is routine.

\section{Conclusion and open problems}

Structured optimization problems, such as the coloring problems considered here, have strong claims with respect to applications. A source of discussion of these applications is the recent dissertation of Marx [24]. It seems interesting and fruitful to consider such problems from the parameterized point of view, and to investigate how such extra problem structure (which tends to increase both computational complexity, and real-world applicability) interacts with parameterizations (such as bounded treewidth), that frequently lead to tractability.

The outcome of the investigation here of some well-known locally or globally constrained coloring problems has turned up a few surprises: first of all, that the LIST CHROMATIC NuMBER problem is actually FPT, when we parameterize by treewidth. It is also somewhat surprising that this good news does not extend to LIST ColoRING, PRECOLORING ExTENSION or EQUITABLE CoLORING, all of which turn out to be hard for $W[1]$. Results of the preliminary version of this paper [13] have led to thorough investigations of structural parameterizations like treewidth or clique-width $[9,16,17,27]$. 
There are many interesting open problems concerning the parameterized complexity of "more structured" combinatorial optimization problems on graphs, parameterized by treewidth. We mention the following:

1. Is the List Edge Chromatic Number problem fixed-parameter tractable, parameterized by treewidth?

2. One can formulate a "list analogue" of the HAMiLToniAn PATH problem as follows: each vertex is assigned a list that is a subset of $\{1,2, \ldots, n\}$ indicating the positions in the ordering of the $n$ vertices implicit in a Hamiltonian path that are permitted to the vertex. Is the LIST HAMILTONIAN PATH problem FPT, parameterized by treewidth?

3. Our hardness results do not exclude the possibility of solving List ColORING, Precoloring EXTENSION, or EQUITABLE CoLORING in time $f(t) \cdot n^{o(t)}$ on graphs of treewidth $t$. Is it possible to show that up to some assumption from complexity theory there are no such algorithms?

\section{Acknowledgments}

This research has been supported by the Australian Research Council through the Australian Centre in Bioinformatics. The first author also acknowledges the support provided by a Fellowship to the Institute of Advanced Studies, Durham University, and the support of the Informatics Institute at the University of Bergen during an extended visit. The second author acknowledges the support of the Norwegian Research Council.

\section{References}

[1] N. Alon, Restricted colorings of graphs, in: K. Walker (Ed.), Surveys in Combinatorics 1993, London Math. Soc. Lecture Notes Series, vol. 187, Cambridge University Press, 1993, pp. 1-33.

[2] S. Arnborg, J. Lagergren, D. Seese, Easy problems for tree-decomposable graphs, Journal of Algorithms 12 (1991) 308-340.

[3] H.L. Bodlaender, A tourist guide through treewidth, Acta Cybernetica 11 (1993) 1-21.

[4] H.L. Bodlaender, F.V. Fomin, Equitable colorings of bounded treewidth graphs, Theoretical Computer Science 349 (2005) 22-30.

[5] H.L. Bodlaender, M. Fellows, M. Langston, M.A. Ragan, F. Rosamond, M. Weyer, Quadratic kernelization for convex recoloring of trees, in: Proceedings COCOON 2007, Lecture Notes in Computer Science, vol. 4598, Springer-Verlag, 2007, pp. 86-96.

[6] R.B. Borie, R.G. Parker, C.A. Tovey, Automatic generation of linear-time algorithms from predicate calculus descriptions of problems on recursively generated graph families, Algorithmica 7 (1992) 555-581.

[7] B. Chor, M. Fellows, M.A. Ragan, I. Razgon, F. Rosamond, S. Snir, Connected coloring completion for general graphs: algorithms and complexity, in: Proceedings COCOON 2007, Lecture Notes in Computer Science, vol. 4598, Springer-Verlag, 2007, pp. 75-85.

[8] B. Courcelle, The monadic second-order logic of graphs I: recognizable sets of finite graphs, Information and Computation 85 (1990) $12-75$.

[9] M. Dom, D. Lokshtanov, S. Saurabh, Y. Villanger, Capacitated domination and covering: a parameterized perspective, in: Proceedings IWPEC 2008, Lecture Notes in Computer Science, vol. 5018, Springer-Verlag, 2008, pp. 78-90.

[10] R.G. Downey, M.R. Fellows, Parameterized Complexity, Springer-Verlag, 1999.

[11] P. Erdös, A.L. Rubin, H. Taylor, Choosability in graphs, Congressus Numerantium 26 (1980) 122-157.

[12] J. Flum, M. Grohe, Parameterized Complexity Theory, Springer-Verlag, 2006.

[13] M. Fellows, F.V. Fomin, D. Lokshtanov, F. Rosamond, S. Saurabh, S. Szeider, C. Thomassen, On the complexity of some colorful problems parameterized by treewidth, in: Proceedings COCOA 2007, Lecture Notes in Computer Science, vol. 4616, Springer-Verlag, 2007, pp. 366-377.

[14] M. Fellows, P. Giannopoulos, C. Knauer, C. Paul, F. Rosamond, S. Whitesides, N. Yu, Milling a graph with turn costs: a parameterized complexity perspective, in: Proceedings WG 2010, Lecture Notes in Computer Science, vol. 6410, Springer-Verlag, 2010, pp. 123-134.

[15] M. Fellows, D. Hermelin, F. Rosamond, S. Vialette, On the fixed-parameter intractability and tractability of multiple-interval graph properties, Theoretical Computer Science 410 (2009) 53-61.

[16] F.V. Fomin, P.A. Golovach, D. Lokshtanov, S. Saurabh, Intractability of Clique-width Parameterizations, SIAM Journal on Computing 39 (5) (2010) $1941-1956$.

[17] F.V. Fomin, P.A. Golovach, D. Lokshtanov, S. Saurabh, Algorithmic lower bounds for problems parameterized by Clique-width, Proceedings SODA (2010) 493-502.

[18] S. Gutner, M. Tarsi, Some results on ( $a: b)$-choosability, Discrete Mathematics 309 (2009) 2260-2270.

[19] A. Hajnal, E. Szemerédi, Proof of a conjecture of P. Erdős, in: Combinatorial Theory and its Applications, vol. II, North-Holland, Amsterdam, 1970 , pp. 601-623.

[20] K. Jansen, P. Scheffler, Generalized colorings for tree-like graphs, Discrete Applied Mathematics 75 (1997) 135-155.

[21] T.R. Jensen, B. Toft, Graph Coloring Problems, Wiley Interscience, 1995.

[22] A.V. Kostochka, M.J. Pelsmajer, D.B. West, A list analogue of equitable coloring, Journal of Graph Theory 44 (2003) $166-177$.

[23] J. Kratochvil, Z. Tuza, M. Voigt, New trends in the theory of graph colorings: choosability and list coloring, in: R. Graham (Ed.), et al., Contemporary Trends in Discrete Mathematics (from DIMACS and DIMATIA to the Future), DIMACS Series in Discrete Mathematics and Theoretical Computer Science, vol. 49, AMS, Providence, 1999, pp. 183-197.

[24] D. Marx, Graph coloring with local and global constraints, Department of Computer Science and Information Theory, Ph.D. dissertation, Budapest University of Technology and Economics, 2004.

[25] W. Meyer, Equitable coloring, American Mathematical Monthly 80 (1973) 920-922.

[26] R. Niedermeier, Invitation to Fixed Parameter Algorithms, Oxford University Press, 2006.

[27] M. Samer, S. Szeider, Constraint satisfaction with bounded treewidth revisited, Journal of Computer and System Sciences 76 (2) (2010) 103-114.

[28] C. Thomassen, Every planar graph is 5-choosable, Journal of Combinatorial Theory Series B 62 (1994) $180-181$.

[29] Z. Tuza, Graph colorings with local constraints - a survey, Discussiones Mathematicae - Graph Theory 17 (1997) $161-228$.

[30] V.G. Vizing, Coloring the vertices of a graph in prescribed colors. Diskret. Analiz No. 29 Metody Diskret. Anal. v Teorii Kodov i Shem 1976 , pp. 3-10.

[31] D.R. Woodall, List colourings of graphs, in: J.W.P. Hirschfeld (Ed.), Surveys in Combinatorics 2001, London Math. Soc. Lecture Notes Series, vol. 288, Cambridge University Press, 2001, pp. 269-301. 